\title{
The Local Group Census: planetary nebulae in the spheroidal galaxies NGC 147, NGC 185 and NGC 205^
}

\author{
R. L. M. Corradi ${ }^{1}$, L. Magrini ${ }^{2}$, R. Greimel ${ }^{1}$, M. Irwin $^{3}$, P. Leisy ${ }^{1,4}$, D. J. Lennon ${ }^{1}$, A. Mampaso ${ }^{4}$, M. Perinotto ${ }^{2}$, \\ D. L. Pollacco ${ }^{5}$, J. R. Walsh ${ }^{6}$, N. A. Walton ${ }^{3}$, and A. A. Zijlstra ${ }^{7}$ \\ ${ }^{1}$ Isaac Newton Group of Telescopes, Apartado de Correos 321, 38700 Santa Cruz de La Palma, Spain \\ e-mail: rcorradi@ing.iac.es \\ 2 Dipartimento di Astronomia e Scienza dello Spazio, Universitá di Firenze, L.go E. Fermi 2, 50125 Firenze, Italy \\ 3 Institute of Astronomy, University of Cambridge, Madingley Road, Cambridge CB3 OHA, UK \\ 4 Instituto de Astrofísica de Canarias, c. vía Láctea s/n, 38200, La Laguna, Tenerife, Spain \\ 5 APS Division, Dept. of Pure and Applied Physics, Queen's University Belfast, Belfast BT7 9NN, Northern Ireland, UK \\ ${ }^{6}$ ST-ECF, ESO, Karl-Schwarzschild-Strasse 2, 85748 Garching bei München, Germany \\ 7 Physics Department, UMIST, PO Box 88, Manchester M60 1QD, UK
}

Received 19 July 2004 / Accepted 16 October 2004

\begin{abstract}
In the framework of our narrow-band survey of Local Group galaxies, we present the results of the search for planetary nebulae (PNe) in three spheroidal companions to the Andromeda galaxy. We find 9 candidate PNe in NGC 147, 5 in NGC 185, and 75 in the $\sim 0.4$ square degree area searched around NGC 205, increasing the number of PNe known in these galaxies. It is shown that in the crowded regions of these galaxies continuum-subtracted images are more effective in detecting $\mathrm{PNe}$ than colour-colour diagrams obtained via automatic photometry.

For NGC 205, the degree of contamination of PNe belonging to the halo of M 31 is estimated; taking it into account, 35 PNe within 1.5 tidal radii from the centre of NGC 205 have been used to build its PN luminosity function.

Candidate PNe in NGC 185 are systematically brighter than those in NGC 147. Considering that star formation is thought to have been much stronger in NGC 185 than in NGC 147 in the last $3 \mathrm{Gyr}$, this might suggest that the bright end of the PN luminosity function is populated by relatively massive stars, as predicted by some recent theoretical models. This result, however, has to be taken with some caution, given the small PN population size of these galaxies and a rather incomplete knowledge of their star formation history.
\end{abstract}

Key words. ISM: planetary nebulae: general - galaxies: stellar content

\section{Introduction}

Planetary nebulae (PNe) are valuable tracers of any stellar population of low and intermediate mass. Owing to their selective emission in a small number of strong and narrow emission lines, they can be discovered at significant distances within the nearby Universe. Their study provides accurate information on the luminosity, age, metallicity, and dynamics of the stellar population from which they derive; this makes them very useful to test a number of theories about the evolution of stars and galaxies.

In order to investigate these issues, the first step is to detect a representative sample of extragalactic PNe. With this aim, we have carried out a narrow- and broad-band imaging survey aimed at cataloguing all classes of emission-line

* Based on observations obtained at the $2.5 \mathrm{~m}$ INT telescope operated on the island of La Palma by the Isaac Newton Group in the Spanish Observatorio del Roque de Los Muchachos of the Instituto de Astrofísica de Canarias. objects in most of the Local Group (LG) galaxies visible from La Palma. The project is called the Local Group Census (LGC), and details of its status can be found at http://www.ing.iac.es/ rcorradi/LGC. So far, the analysis of the LGC data has been focussed on the search for PNe in dwarf irregular galaxies, and resulted in a significant increase of the total population of PNe known in these systems (Magrini et al. 2002, 2003, 2004; Leisy et al. 2004).

The most common morphological type in the LG is however that of the so-called spheroidal ( $\mathrm{Sph}$ ) galaxies. Most objects in this class are dwarf galaxies, and their distribution in the LG, total number and origin is still not well understood (see e.g. Van den Berg 2000, hereafter vdB00). NGC 147, NGC 185 and NGC 205 are the most luminous Sph galaxies of the LG, and owing to their relatively high luminosity they should not be be considered as dwarfs. They are all associated with the Andromeda Galaxy. NGC 205 is fully embedded in the halo of M 31, and its outer isophotes are clearly distorted by tidal interaction with the large spiral galaxy. NGC 147 and NGC 185 
Table 1. Log of the INT+WFC observations.

\begin{tabular}{|c|c|c|c|c|c|}
\hline \multirow{2}{*}{$\begin{array}{l}\text { Galaxy } \\
\text { NGC } 147\end{array}$} & \multicolumn{2}{|c|}{$\begin{array}{c}\text { Field centre } \\
\text { RA (J2000) Dec }\end{array}$} & \multirow{2}{*}{$\begin{array}{c}\text { Filters } \\
{[\mathrm{O} \text { III], } \mathrm{H} \alpha}\end{array}$} & \multirow{2}{*}{$\begin{array}{l}\text { Exp. time } \\
1200 \mathrm{~s} \times 3\end{array}$} & \multirow{2}{*}{$\begin{array}{c}\text { Date } \\
2003-08-23\end{array}$} \\
\hline & 03300.0 & 483024.76 & & & \\
\hline & & & $g^{\prime}, r^{\prime}$ & $400 \mathrm{~s} \times 3$ & \\
\hline \multirow[t]{3}{*}{ NGC 185} & 03857.4 & 482014.36 & [O III], $\mathrm{H} \alpha$ & $1200 \mathrm{~s} \times 3$ & 2003-09-05 \\
\hline & & & Strömgren $y$ & $600 \mathrm{~s} \times 3$ & \\
\hline & & & $g^{\prime}, r^{\prime}$ & $400 \mathrm{~s} \times 3$ & \\
\hline \multirow[t]{4}{*}{ NGC 205A } & 04010.0 & 413612.98 & {$[\mathrm{O} \mathrm{III}], \mathrm{H} \alpha$} & $1200 \mathrm{~s} \times 3$ & $2003-09-16$ \\
\hline & & & Strömgren $y$ & $600 \mathrm{~s} \times 3$ & \\
\hline & & & $r^{\prime}$ & $400 \mathrm{~s} \times 3$ & \\
\hline & & & $g^{\prime}$ & $400 \mathrm{~s} \times 3$ & $2003-08-16$ \\
\hline \multirow[t]{4}{*}{ NGC 205B } & 04030.0 & 414200.36 & [O III], $\mathrm{H} \alpha$ & $1200 \mathrm{~s} \times 3$ & $2003-09-16$ \\
\hline & & & Strömgren $y$ & $600 \mathrm{~s} \times 3$ & \\
\hline & & & $r^{\prime}$ & $400 \mathrm{~s} \times 3$ & \\
\hline & & & $g^{\prime}$ & $400 \mathrm{~s} \times 3$ & $2003-08-16$ \\
\hline
\end{tabular}

form a pair at an apparent distance of 12 degrees from M 31, very close to the projected centre of mass of the LG. Prior to our work, 5 candidate PNe were detected in NGC 185 (Ford et al. 1973, 1977), 5 in NGC 147 (Ford et al. 1977), and 28 in NGC 205 (Ford et al. 1973; Ciardullo et al. 1989). In this paper we present new [O III], $\mathrm{H} \alpha$, and "continuum" images of these three galaxies. These allowed us to detect 9 candidate $\mathrm{PNe}$ in NGC 147, 5 in NGC 185, and 75 in the $\sim 0.4$ square degree area searched around NGC 205.

\section{Observations}

The LGC makes use of the prime focus wide field camera (WFC) of the $2.54 \mathrm{~m}$ Isaac Newton Telescope (INT) at La Palma. The detector of the WFC is composed of four thinned EEV42 CCDs with $2048 \times 4096$ pixels each, with a sampling of $0{ }^{\prime} 33$ per pixel, resulting in a total field of view of $34^{\prime} \times 34^{\prime}$. The filters used for the images considered in this paper are: [O III] (with central wavelength and full width at half maximum of $500.8 \mathrm{~nm}$ and $10.0 \mathrm{~nm}$, respectively), $\mathrm{H} \alpha$ (656.8/9.5), Strömgren $y$ (550.5/24.0), as well as the broad band $g^{\prime}$ and $r^{\prime}$ filters of the Sloan photometric system. The Strömgren $y, g^{\prime}$ and $r^{\prime}$ images were used to evaluate the continuum emission in close proximity to the [O III] and $\mathrm{H} \alpha$ wavelengths. Note that the $\mathrm{H} \alpha$ filter includes the contribution of the [N II] doublet at $654.8 \mathrm{~nm}$ and $658.3 \mathrm{~nm}$; for the sake of conciseness, in the following we label data in this filter only with the symbol "H $\alpha$ ".

While one WFC field was observed for NGC 147 and NGC 185, two overlapping adjacent fields were observed for NGC 205 owing to its larger size and in order to estimate the degree of contamination of PNe belonging to M 31. These overlapping fields cover a field of $\sim 40^{\prime} \times 40^{\prime}$. The log of the observations is presented in Table 1 . Seeing ranged from 1.1 to 1.5 arcsec.

Flux calibration of the broad and narrowband images were done by observing the standard photometric stars SA 95-42, SA 95-190, and SA 92-263, the spectrophotometric star G 191-B2B, and the Galactic PN M 2-1, during photometric nights.

\section{Data reduction and analysis}

Data was reduced using routines available within $\mathrm{IRAF}^{1}$. The frames were de-biased, flat-fielded, and linearity-corrected using the ING WFC data-reduction pipeline (Irwin \& Lewis 2001). The instrumental magnitudes for the narrowband filters were calibrated by convolving the spectrum of the spectrophotometric standard star (Oke 1990) or the PN M 2-1 (Wright et al. 2004) with the response curve of each filter, computed for the $\mathrm{f} / 3.3$ converging beam of the INT prime focus. Astrometric solutions were computed using tasks CCMAP and CCTRAN and the USNO A2.0 catalogue (Monet et al. 1998). The accuracy of astrometry is $\sim 0 ! 3 \mathrm{rms}$. In order to detect PNe, images were then analysed using the following two methods.

\subsection{Continuum-subtracted images}

Following the procedure described in Magrini et al. (2002, 2003), [O III] and $\mathrm{H} \alpha$ continuum-subtracted images were obtained. First, all images were corrected for geometrical distortions and aligned to the [O III] frame. The $r^{\prime}$ images were then used as the reference continuum for the $\mathrm{H} \alpha$ frames, and after proper scaling they were subtracted from them. For the continuum subtraction of the [O III] images, we have used the $g^{\prime}$ frames for NGC 185 and NGC 205, and the Strömgren $y$ image for NGC 147. In the continuum-subtracted frames, candidate PNe were identified as point-like sources (PNe cannot be resolved at the distance of our target galaxies) emitting in [O III], although in most cases they were also detected in $\mathrm{H} \alpha$.

\footnotetext{
${ }^{1}$ IRAF is distributed by the National Optical Astronomy Observatories, which is operated by the Association of Universities for Research in Astronomy, Inc. (AURA) under cooperative agreement with the National Science Foundation.
} 
Emission-line fluxes were measured in the continuumsubtracted frames, using the IRAF task APPHOT. They were corrected for the contribution of the [O III] and $\mathrm{H} \alpha$ lines in the $g^{\prime}$ and $r^{\prime}$ continuum images, respectively. For the [O III] line, fluxes were also corrected for the contribution in the WFC filter of the companion oxygen line at $\lambda=495.9 \mathrm{~nm}$ (considering the systemic radial velocity of each galaxy), and transformed to equivalent $V$ magnitudes via the formula $\left.m_{[\mathrm{O}} \mathrm{III}\right]=$ $-2.5 \log F_{[\mathrm{O} \text { III] }}-13.74$ (Jacoby 1989). The errors of the [O III] and $\mathrm{H} \alpha$ fluxes of the candidate $\mathrm{PNe}$, including photon statistics, background and flux calibration uncertainties, amount to some $10 \%$ for fluxes larger than $3.2 \times 10^{-15} \mathrm{erg} \mathrm{cm}^{-2} \mathrm{~s}^{-1}\left(m_{[\mathrm{O} \mathrm{IIII}]}=\right.$ 22.5 ), to $20-30 \%$ for fluxes up to $8.0 \times 10^{-16} \mathrm{serg} \mathrm{cm}^{-2} \mathrm{~s}^{-1}$ $\left(m_{[\mathrm{O} \mathrm{III}]}=24.0\right)$, and are larger for the faintest lines.

\subsection{Colour-colour diagrams}

Automatic point-spread-function (PSF) photometry in all filters for all the observed fields were performed using the packages DAOPHOT/ALLSTAR (Stetson 1994). First, point-like sources were searched using a detection threshold $6 \sigma$ over the background. Then the PSF was built using some forty well exposed and isolated stars in each CCD frame. In very crowded fields, a simple Gaussian PSF was first computed in order to subtract objects close to all the stars that were used to build the final PSF. This procedure was iterated increasing the degree of the polynomials that describe the spatial variations of the PSF throughout the field. Once built the PSF, instrumental magnitudes were measured for all unresolved sources in the various filters.

Different kind of colour-colour diagrams were then produced. A priori, the most obvious choice to highlight emission-line objects would be to consider an $\mathrm{H} \alpha-r^{\prime}$ vs. [O III $]-g^{\prime}$ diagram. An example is given in Fig. 1 (upper panel) for the two WFC fields of NGC 205. In this diagram, photometry was obtained for 71500 stars, and candidate PNe selected in the continuum-subtracted images clearly show up as objects with generally strong [O III] and $\mathrm{H} \alpha$ excesses (large dots) with respect to normal stars (small dots). However, using this diagram some $50 \%$ of the candidate PNe were missed because in the crowded inner regions of the galaxy it is not possible to measure their $r^{\prime}$ magnitude (even with a lower detection threshold). We have also considered the less "natural" $\mathrm{H} \alpha-g^{\prime}$ vs. [O III] $-g^{\prime}$ diagram (Fig. 1, lower panel), because in the $g^{\prime}$ band the galaxy stellar background is fainter than in $r^{\prime}$, as red stars dominate the overall emission of these relatively old galaxies, and thus PNe are more easily detected. In such a diagram, the detection rate of $\mathrm{PNe}$ increases to $75 \%$, but the degree of confusion between PNe and stars also increases. The search is improved if an additional list of objects which are detected by PSF photometry in the [O III] and $\mathrm{H} \alpha$ frames, but not in the broadband ones, is produced; these sources should be checked individually in the original images to remove spurious detections in the narrowband filters. Even so, however, a few PNe are missed.

We conclude that, as expected, in the crowded regions of galaxies the visual inspection of continuum-subtracted images,
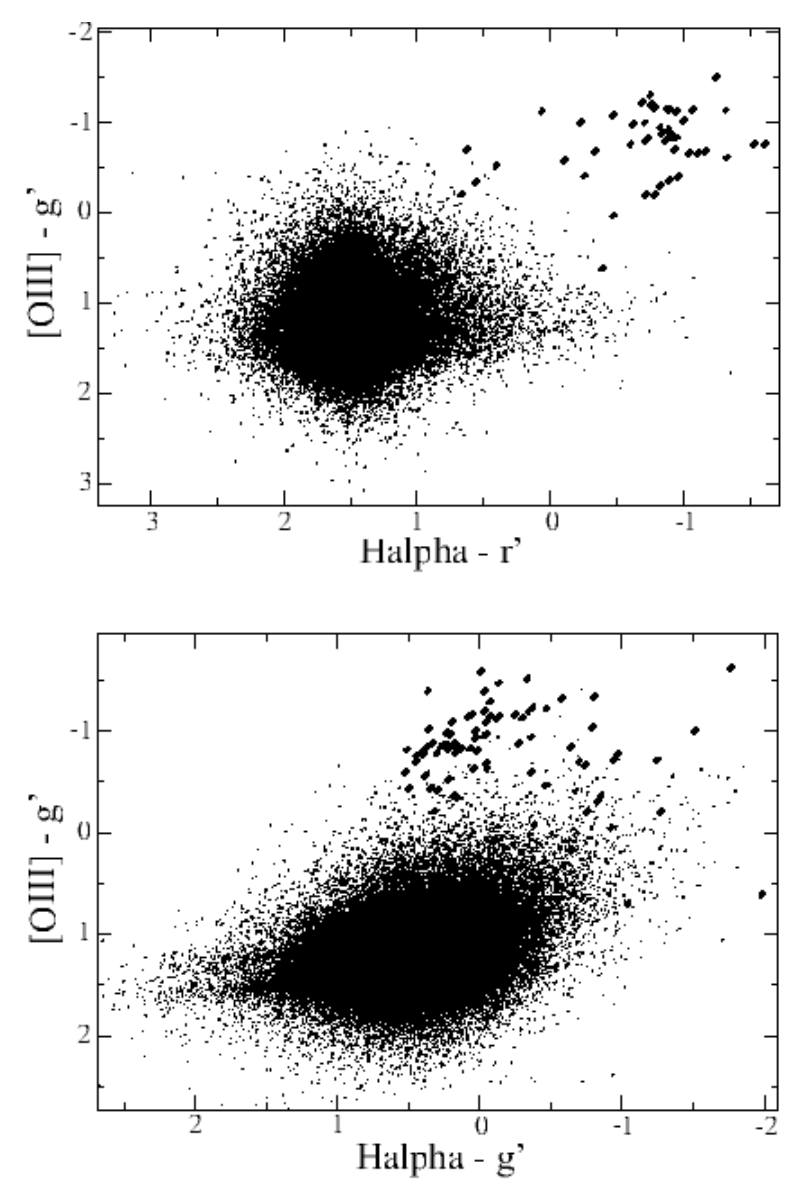

Fig. 1. Colour-colour diagrams for the two WFC fields around NGC 205. Colours are computed from instrumental magnitudes, so that the zero points of the $x$ and $y$ axes are arbitrary.

although somewhat subjective and time consuming, is more effective in detecting PNe than the analysis of colour-colour diagrams obtained via PSF photometry. Even so, the use of these diagrams remains a valid complementary tool to double check identifications of candidate $\mathrm{PNe}$, verifying for example that that no emission line sources have been missed in the visual inspection of the large fields observed. In uncrowded regions, colour-colour diagrams are instead a powerful automatic tool to detect PNe, as shown by Arnaboldi et al. (2003) for the external regions of galaxies and the intergalactic space. Note that other authors (e.g. Feldmeier et al. 2003) apply automated detection algorithms directly to the continuum-subtracted frames in order to detect extragalactic PNe.

\section{Results}

\subsection{NGC 147}

Nine candidate PNe were identified in the continuumsubtracted images of NGC 147 and colour-colour diagrams. They are listed in Table 2 with their coordinates, [OIII] and $\mathrm{H} \alpha$ fluxes, and $\left.\mathrm{m}_{[\mathrm{O}} \mathrm{III}\right]$. Their positions in the sky are indicated by crosses in Fig. 2. They are all located inside the $m_{p g}=26.5 \mathrm{mag} \operatorname{arcsec}^{-2}$ isophote of NGC $147\left(12^{\prime} \times 18^{\prime}\right.$, Holmberg 1958). Two of our candidate PNe (id.n. 4 and 7) 


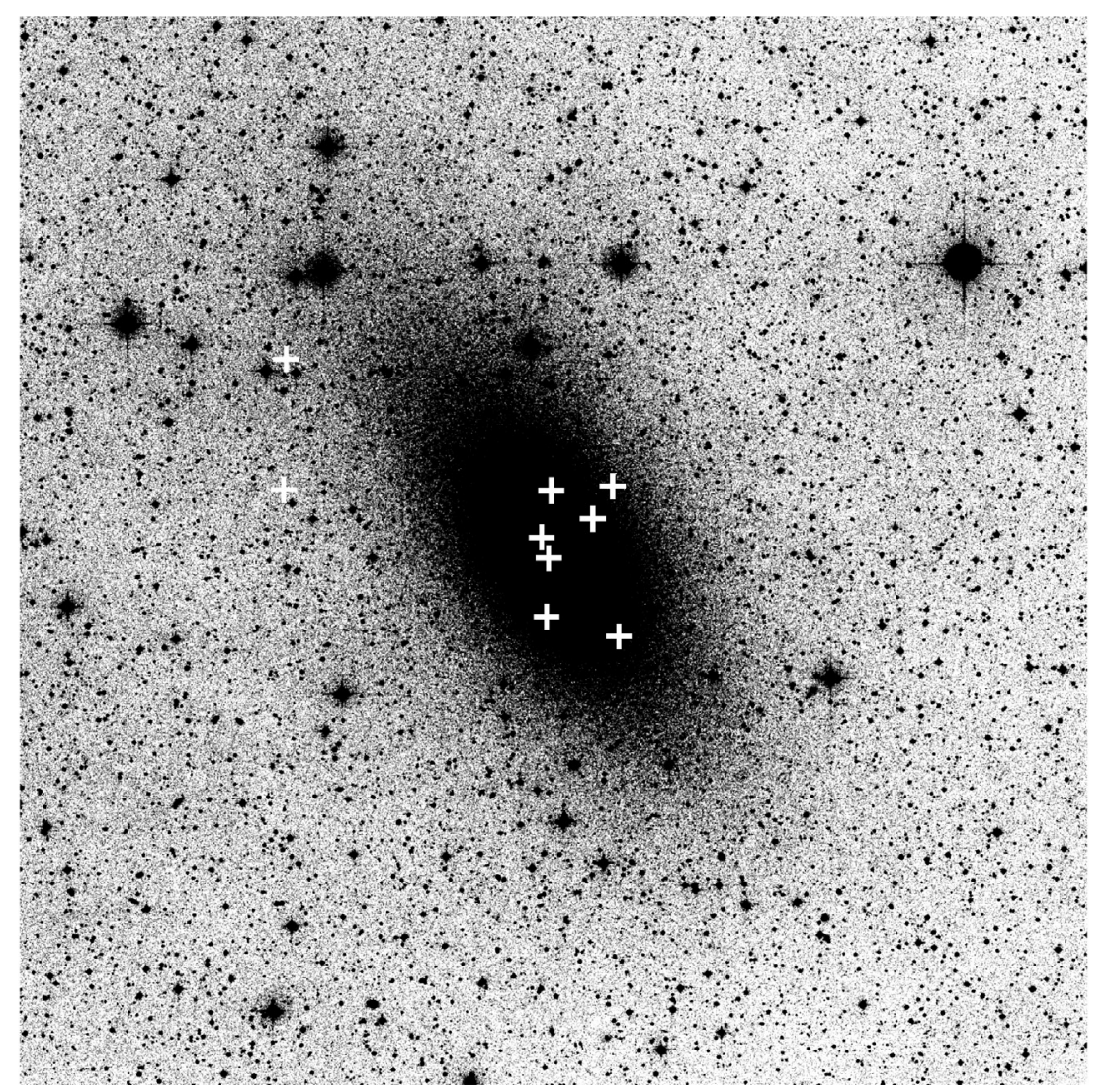

Fig. 2. Digitized Sky Survey image of an area of $34^{\prime} \times 34^{\prime}$ around NGC 147. North is at the top, East to the left. Candidate PNe are marked with crosses.

Table 2. PN candidates in NGC 147. Observed [O III]500.7 and $\mathrm{H} \alpha$ fluxes are given in $10^{-16} \mathrm{erg} \mathrm{cm}^{-2} \mathrm{~s}^{-1} . F_{[\mathrm{OIII}]}$ are corrected for the contribution of the companion oxygen line at $495.9 \mathrm{~nm}$.

\begin{tabular}{rccccc}
\hline \hline Id. & \multicolumn{2}{c}{ RA (2000.0) Dec } & $F_{[\mathrm{OIII}]}$ & $F_{\mathrm{H} \alpha}$ & $\mathrm{m}_{[\mathrm{O} \mathrm{III]}}$ \\
\hline 1 & $0: 32: 59.97$ & $48: 32: 26.0$ & 2.63 & 1.71 & 25.21 \\
2 & $0: 33: 03.78$ & $48: 31: 25.2$ & 11.7 & 4.14 & 23.59 \\
3 & $0: 32: 58.79$ & $48: 27: 40.5$ & 13.8 & 8.99 & 23.41 \\
4 & $0: 33: 12.66$ & $48: 28: 18.1$ & 31.8 & 37.3 & 22.50 \\
5 & $0: 33: 12.29$ & $48: 30: 09.3$ & 3.05 & - & 25.05 \\
6 & $0: 33: 13.63$ & $48: 30: 49.4$ & 0.83 & 5.77 & 26.46 \\
7 & $0: 33: 11.76$ & $48: 32: 17.8$ & 31.1 & 12.1 & 22.53 \\
8 & $0: 34: 03.25$ & $48: 32: 18.3$ & 10.4 & 0.41 & 23.72 \\
9 & $0: 34: 02.87$ & $48: 36: 28.0$ & 7.29 & 3.55 & 24.10 \\
\hline
\end{tabular}

correspond to sources n. 1 and 4, respectively, detected by Ford et al. (1977). The other three objects listed by Ford et al. (1977) were found to be stars as they show a significant continuum emission.

\subsection{NGC 185}

In this case, we confirm the five candidate PNe originally discovered by Ford et al. (1977), which include one H $\alpha$ source detected by Martinez-Delgado et al. (1999), while the additional source found by the latter authors does not emit in [O III], and
Table 3. As in Table 2, but for NGC 185.

\begin{tabular}{rccccc}
\hline \hline Id. & \multicolumn{2}{c}{ RA (2000.0) Dec } & $F_{[\mathrm{OIII}]}$ & $F_{\mathrm{H} \alpha}$ & $\mathrm{m}_{[\mathrm{O} \mathrm{III}]}$ \\
\hline 1 & $0: 38: 53.10$ & $48: 20: 07.9$ & 105 & 52.6 & 21.21 \\
2 & $0: 38: 57.28$ & $48: 20: 10.4$ & 132 & 13.1 & 20.96 \\
3 & $0: 39: 00.23$ & $48: 19: 47.3$ & 79.7 & 30.0 & 21.51 \\
4 & $0: 38: 56.40$ & $48: 19: 21.4$ & 235 & 64.0 & 20.33 \\
5 & $0: 38: 47.94$ & $48: 19: 31.9$ & 27.7 & 5.94 & 22.66 \\
\hline
\end{tabular}

thus was conservatively left out from our list of candidate PNe. This is presented in Table 3, and PNe are indicated by crosses in Fig. 3. All objects are located in the bright, innermost regions of the galaxy, well inside its optical size $\left(12^{\prime} \times 13^{\prime}\right.$, Holmberg 1958). The [O III] magnitudes that we have determined are in good agreement with those measured by Ciardullo et al. (1989), with the exception of PN n. 2 which we measured to be $\sim 0.5$ mag brighter. This is probably due to its location in the very central and crowded regions of the galaxy, which makes it difficult to derive its luminosity in the observations of Ciardullo et al. (1989), as the detector pixel projected in the sky was relatively big $\left(0^{\prime \prime} .86\right)$ considering the rapidly varying galaxy background. Our images also confirm the presence of a diffuse ionized medium emitting both in $\mathrm{H} \alpha$ and [O III] in a region of $\sim 60^{\prime \prime} \times 60^{\prime \prime}$ in the centre of the galaxy (e.g. Martinez-Delgado et al. 1999). 


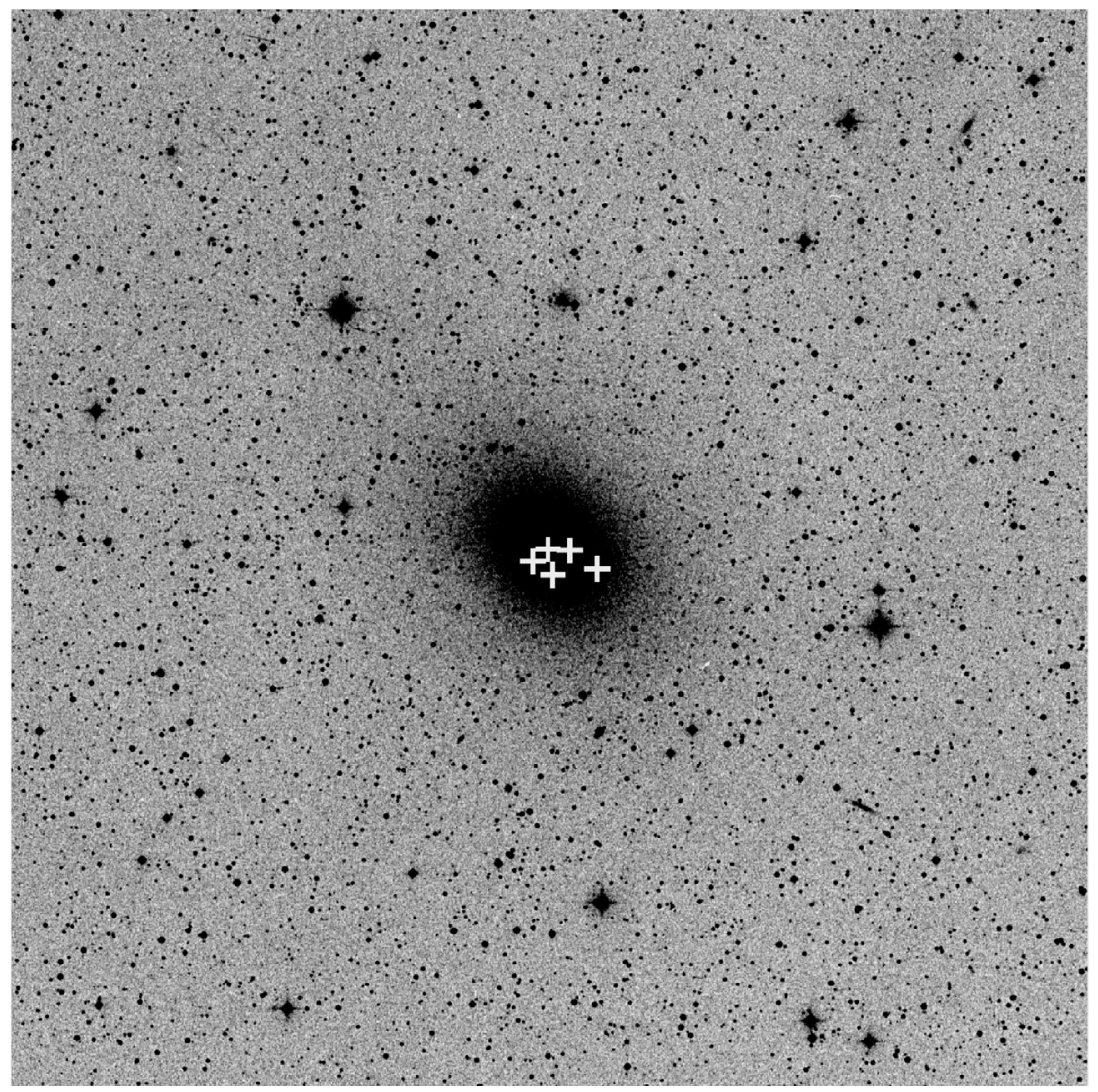

Fig. 3. Digitized Sky Survey image of an area of $34^{\prime} \times 34^{\prime}$ around NGC 185 . North is at the top, East to the left. Candidate PNe are marked with crosses.

\subsection{NGC 205}

Our narrowband images allowed us to identify $75 \mathrm{PNe}$ in the $\sim 0.4$ square degree area searched around NGC 205. Forty of them are located within the $m_{p g}=26.5 \mathrm{mag} \operatorname{arcsec}^{-2}$ isophote $\left(16^{\prime} \times 26^{\prime}\right.$, Holmberg 1958), and the remaining ones outside it. The candidates are listed in Table 4, and their positions within NGC 205 indicated in Fig. 4.

Previous survey for PNe in this galaxy led to the discovery of $24 \mathrm{PNe}$ (Ford et al. 1977; Ciardullo et al. 1989). We have recovered 20 of them, while another 3 were found to be stars (PNe 12, 21, and 28 in Table 6 of Ciardullo et al. 1989), and we did not detect their PN n.14. Outside NGC 205, ten PNe in our list were previously found by Ford \& Jacoby (1978) and Meyssonnier et al. (1993). Merrett et al. (2003) included the region around NGC 205 in their survey for PNe in M 31, but in their paper they do not specifically discuss the results for this area.

A simple visual inspection of the distribution of PNe in Fig. 4 suggests that a number of PNe must be associated with the disk and halo of M 31. A precise calculation of the amount of contamination of M $31 \mathrm{PNe}$ at the location of NGC 205 is not easy, as it depends on the (spatially variable) completeness of our search in addition to the (also varying) contribution from the disk and halo of M 31. A first-order estimate was obtained in the following way. In our images, we have masked an elliptical region centred on NGC 205 and extending out to 1.5 (9!3, Mateo 1998) and 2 tidal radii. Then the density of PNe was estimated in stripes parallel to M 31's major axis, each with a width of 4 arcmin, and excluding the masked region. Except for the first strip, likely dominated by the disk of M 31, there is a shallow decrease of the density of PNe with distance from M 31 (Fig. 5), which allows us to adopt an average figure of $2.3 \times 10^{-2} \mathrm{PNe} \operatorname{arcmin}^{-2}$ belonging to M 31 in this area and at the detection limit of our survey. This would mean that, among the $27 \mathrm{PNe}$ inside one tidal radius of NGC 205, only one or two might belong to $\mathrm{M} 31$, being seen in projection over its spheroidal companion. Contamination by M 31's PNe would become significant only outside 1.5 tidal radii, which is the "borderline" that we adopt (see the ellipse in Fig. 4) as the limit at which we are confident that nearly all the 35 candidate $\mathrm{PNe}$ detected inside it belong in fact to NGC 205.

Consistent results are obtained if M 31's contamination is estimated from the surface brightness photometry of the halo of M 31 (Irwin et al., in preparation). From this, a bolometric luminosity of $6 \times 10^{7} L_{\odot}$ is computed for the halo light integrated within 1.5 tidal radii of NGC 205 . This can be transformed into a total number of PNe using the theoretical expectations of stellar evolution theories (Renzini \& Buzzoni 1986). Adopting $\log \alpha=\log \left(N_{\mathrm{PN}} / L_{\mathrm{bol}}\right)=-6.5$ (Buzzoni \& Arnaboldi 2004), this gives a total of 19 PNe belonging to M 31 and located within 1.5 tidal radii of NGC 205 . Correcting this number for the completeness limit of our survey (see next section) using the empirical law of the PN luminosity function (PNLF, Ciardullo et al. 1989), it implies that we should have detected three or four of M 31's PNe in the area of NGC 205, in very 
Table 4. As in Table 2, but for NGC 205. The last column indicates whether the candidate PNe are inside ("i") or outside ("o") 1.5 tidal radii of the galaxy.

\begin{tabular}{rcccccc}
\hline \hline Id. & RA $(2000)$ Dec & $F_{[\mathrm{OIII}]}$ & $F_{\mathrm{H} \alpha}$ & $\mathrm{m}_{[\mathrm{O} \text { III] }}$ & \\
\hline 1 & $0: 38: 28.48$ & $41: 28: 36.9$ & 9.19 & 4.09 & 23.85 & $\mathrm{o}$ \\
2 & $0: 38: 46.16$ & $41: 28: 19.4$ & 161 & 62.5 & 20.74 & $\mathrm{o}$ \\
3 & $0: 38: 48.50$ & $41: 39: 37.0$ & 33.5 & 13.9 & 22.45 & $\mathrm{o}$ \\
4 & $0: 39: 10.28$ & $41: 43: 16.5$ & 7.94 & 2.40 & 24.01 & $\mathrm{o}$ \\
5 & $0: 39: 20.14$ & $41: 43: 23.0$ & 0.63 & 0.01 & 26.75 & $\mathrm{o}$ \\
6 & $0: 39: 34.87$ & $41: 23: 44.4$ & 9.81 & 3.81 & 23.78 & $\mathrm{o}$ \\
7 & $0: 39: 38.62$ & $41: 21: 00.4$ & 3.45 & 2.94 & 24.92 & $\mathrm{o}$ \\
8 & $0: 39: 49.23$ & $41: 24: 09.7$ & 40.5 & 47.7 & 22.24 & $\mathrm{o}$ \\
9 & $0: 39: 50.69$ & $41: 30: 42.8$ & 22.0 & 5.79 & 22.91 & $\mathrm{o}$ \\
10 & $0: 39: 51.22$ & $41: 27: 07.4$ & 6.50 & 6.90 & 24.23 & $\mathrm{o}$ \\
11 & $0: 39: 55.45$ & $41: 23: 25.6$ & 6.78 & 4.91 & 24.18 & $\mathrm{o}$ \\
12 & $0: 39: 56.59$ & $41: 35: 18.8$ & 24.1 & 6.61 & 22.80 & $\mathrm{o}$ \\
13 & $0: 39: 56.87$ & $41: 43: 06.7$ & 51.9 & 15.6 & 21.97 & $\mathrm{i}$ \\
14 & $0: 39: 59.47$ & $41: 46: 16.8$ & $f a i n t$ & 41.1 & - & $\mathrm{i}$ \\
15 & $0: 40: 00.51$ & $41: 49: 19.2$ & 52.3 & 18.5 & 21.96 & $\mathrm{o}$ \\
16 & $0: 40: 02.75$ & $41: 42: 13.9$ & 7.24 & 10.3 & 24.11 & $\mathrm{i}$ \\
17 & $0: 40: 03.38$ & $41: 43: 59.8$ & 23.4 & 37.7 & 22.84 & $\mathrm{i}$ \\
18 & $0: 40: 08.08$ & $41: 45: 24.3$ & 4.53 & 0.60 & 24.62 & $\mathrm{i}$ \\
19 & $0: 40: 08.88$ & $41: 40: 43.7$ & 94.4 & 43.6 & 21.32 & $\mathrm{i}$ \\
20 & $0: 40: 10.47$ & $41: 49: 18.8$ & 11.3 & 4.60 & 23.63 & $\mathrm{i}$ \\
21 & $0: 40: 10.60$ & $41: 21: 23.9$ & 26.6 & 6.84 & 22.70 & $\mathrm{o}$ \\
22 & $0: 40: 11.18$ & $41: 40: 47.9$ & 8.35 & 3.14 & 23.96 & $\mathrm{i}$ \\
23 & $0: 40: 12.11$ & $41: 45: 32.1$ & 14.2 & 25.1 & 23.38 & $\mathrm{i}$ \\
24 & $0: 40: 13.57$ & $41: 38: 41.8$ & 158 & 49.2 & 20.76 & $\mathrm{i}$ \\
25 & $0: 40: 14.57$ & $41: 37: 42.0$ & 117 & 39.0 & 21.09 & $\mathrm{i}$ \\
26 & $0: 40: 15.06$ & $41: 42: 02.2$ & 4.86 & 2.97 & 24.54 & $\mathrm{i}$ \\
27 & $0: 40: 15.80$ & $41: 28: 22.9$ & 9.53 & 7.50 & 23.81 & $\mathrm{o}$ \\
28 & $0: 40: 17.48$ & $41: 45: 59.0$ & 19.4 & 11.0 & 23.04 & $\mathrm{i}$ \\
29 & $0: 40: 17.58$ & $41: 38: 40.4$ & 6.50 & 0.83 & 24.23 & $\mathrm{i}$ \\
30 & $0: 40: 17.98$ & $41: 38: 32.6$ & 89.1 & 36.6 & 21.38 & $\mathrm{i}$ \\
31 & $0: 40: 18.70$ & $41: 41: 42.8$ & 1.87 & 10.5 & 25.58 & $\mathrm{i}$ \\
32 & $0: 40: 19.27$ & $41: 41: 47.4$ & 2.54 & 13.5 & 25.25 & $\mathrm{i}$ \\
33 & $0: 40: 19.94$ & $41: 38: 24.3$ & 84.7 & 28.4 & 21.44 & $\mathrm{i}$ \\
34 & $0: 40: 20.36$ & $41: 38: 17.3$ & 101 & 44.6 & 21.25 & $\mathrm{i}$ \\
35 & $0: 40: 20.45$ & $41: 38: 43.6$ & 17.8 & 8.42 & 23.13 & $\mathrm{i}$ \\
36 & $0: 40: 21.00$ & $41: 41: 42.0$ & 15.5 & 18.6 & 23.28 & $\mathrm{i}$ \\
37 & $0: 40: 21.26$ & $41: 38: 38.5$ & 26.4 & 44.1 & 22.70 & $\mathrm{i}$ \\
38 & $0: 40: 21.53$ & $41: 42: 26.1$ & 71.1 & 40.5 & 21.63 & $\mathrm{i}$ \\
\hline & & & & & &
\end{tabular}

good agreement with our results from the PN counts (also estimating 3 contaminating PNe within 1.5 tidal radii). Note, however, that the value of $\alpha$ can vary by more than one order of magnitude depending on the galaxy type, and the value to be used for the halo of M 31 is uncertain as the population of the PN progenitors is not well known.

\subsection{Completeness limit and population size}

The completeness limit of our search for PNe was computed as in Magrini et al. (2002, 2003). We estimated the number of "missing" PNe by adding "artificial stars" with various $\left.\mathrm{m}_{[\mathrm{O}} \mathrm{III}\right]$ in both narrowband and continuum images, and then computing the recovery rate of such artificial objects. As the galaxy

\begin{tabular}{ccccccc}
\hline \hline Id. & RA $(2000.0)$ Dec & $F_{\text {[OIII] }}$ & $F_{\mathrm{H} \alpha}$ & $\mathrm{m}_{[\mathrm{O} \mathrm{III]}}$ & \\
\hline 39 & $0: 40: 23.16$ & $41: 28: 11.3$ & 1.27 & - & 26.00 & $\mathrm{o}$ \\
40 & $0: 40: 23.48$ & $41: 37: 35.4$ & 7.28 & - & 24.10 & $\mathrm{i}$ \\
41 & $0: 40: 24.78$ & $41: 37: 27.7$ & 68.8 & 30.9 & 21.67 & $\mathrm{i}$ \\
42 & $0: 40: 25.53$ & $41: 40: 06.5$ & 31.8 & 32.8 & 22.50 & $\mathrm{i}$ \\
43 & $0: 40: 26.26$ & $41: 20: 27.7$ & 159 & 57.1 & 20.76 & $\mathrm{o}$ \\
44 & $0: 40: 28.31$ & $41: 39: 16.1$ & 20.2 & 12.9 & 23.00 & $\mathrm{i}$ \\
45 & $0: 40: 28.81$ & $41: 33: 00.6$ & 26.6 & 75.6 & 22.70 & $\mathrm{i}$ \\
46 & $0: 40: 32.26$ & $41: 39: 02.8$ & 23.6 & 7.88 & 22.83 & $\mathrm{i}$ \\
47 & $0: 40: 35.06$ & $41: 49: 33.6$ & 2.40 & 4.92 & 25.31 & $\mathrm{o}$ \\
48 & $0: 40: 35.76$ & $41: 41: 39.9$ & 5.13 & 5.98 & 24.48 & $\mathrm{i}$ \\
49 & $0: 40: 36.32$ & $41: 42: 02.4$ & 29.2 & 26.4 & 22.59 & $\mathrm{i}$ \\
50 & $0: 40: 37.88$ & $41: 23: 30.9$ & 3.45 & 0.08 & 24.92 & $\mathrm{o}$ \\
51 & $0: 40: 40.63$ & $41: 39: 05.5$ & 52.3 & 15.2 & 21.96 & $\mathrm{i}$ \\
52 & $0: 40: 41.29$ & $41: 45: 17.3$ & 8.48 & 4.60 & 23.94 & $\mathrm{i}$ \\
53 & $0: 40: 42.68$ & $41: 29: 38.4$ & 6.98 & 2.28 & 24.15 & $\mathrm{o}$ \\
54 & $0: 40: 43.93$ & $41: 22: 11.0$ & 79.9 & 33.4 & 21.50 & $\mathrm{o}$ \\
55 & $0: 40: 45.42$ & $41: 28: 37.7$ & 4.29 & 0.70 & 24.68 & $\mathrm{o}$ \\
56 & $0: 40: 45.84$ & $41: 43: 36.6$ & 2.43 & 4.69 & 25.30 & $\mathrm{i}$ \\
57 & $0: 40: 47.77$ & $41: 37: 29.3$ & 10.2 & 4.26 & 23.74 & $\mathrm{i}$ \\
58 & $0: 40: 49.57$ & $41: 38: 12.0$ & 13.0 & 40.1 & 23.48 & $\mathrm{i}$ \\
59 & $0: 40: 50.40$ & $41: 23: 35.4$ & 8.15 & 4.72 & 23.98 & $\mathrm{o}$ \\
60 & $0: 40: 54.29$ & $41: 36: 09.2$ & 7.48 & 4.64 & 24.07 & $\mathrm{o}$ \\
61 & $0: 40: 54.65$ & $41: 48: 15.9$ & 66.4 & 30.1 & 21.70 & $\mathrm{o}$ \\
62 & $0: 40: 56.75$ & $41: 19: 31.3$ & 5.99 & 3.56 & 24.32 & $\mathrm{o}$ \\
63 & $0: 40: 58.38$ & $41: 24: 24.3$ & 3.73 & 5.00 & 24.83 & $\mathrm{o}$ \\
64 & $0: 40: 58.76$ & $41: 29: 32.2$ & 140 & 45.3 & 20.89 & $\mathrm{o}$ \\
65 & $0: 41: 01.80$ & $41: 32: 22.5$ & 5.55 & 3.27 & 24.40 & $\mathrm{o}$ \\
66 & $0: 41: 10.74$ & $41: 42: 10.8$ & 13.7 & 18.6 & 23.42 & $\mathrm{o}$ \\
67 & $0: 41: 13.62$ & $41: 27: 53.4$ & 9.81 & 3.10 & 23.78 & $\mathrm{o}$ \\
68 & $0: 41: 15.71$ & $41: 27: 19.7$ & 28.2 & 8.88 & 22.63 & $\mathrm{o}$ \\
69 & $0: 41: 19.08$ & $41: 51: 46.4$ & 23.9 & 15.7 & 22.81 & $\mathrm{o}$ \\
70 & $0: 41: 19.76$ & $41: 41: 09.8$ & 22.0 & 6.65 & 22.91 & $\mathrm{o}$ \\
71 & $0: 41: 21.45$ & $41: 29: 59.1$ & 5.52 & 0.66 & 24.40 & $\mathrm{o}$ \\
72 & $0: 41: 21.59$ & $41: 28: 53.6$ & 7.54 & - & 24.07 & $\mathrm{o}$ \\
73 & $0: 41: 25.40$ & $41: 28: 22.2$ & 15.5 & 4.65 & 23.28 & $\mathrm{o}$ \\
74 & $0: 41: 27.21$ & $41: 33: 13.4$ & 2.37 & 0.79 & 25.32 & $\mathrm{o}$ \\
75 & $0: 41: 28.81$ & $41: 26: 47.8$ & 11.8 & 12.8 & 23.58 & $\mathrm{o}$ \\
& & & & & & \\
\hline & & & & & &
\end{tabular}

background and crowding become progressively stronger toward the central regions of these spheroidal galaxies, we have computed the recovery rate in concentric annuli centred on the galaxy nuclei and with radial steps of 60 arcsec. We find that, in the inner regions of the three galaxies, namely around a radius of 120 arcsec, the recovery rate is $\sim 50 \%$ (this would define the incompleteness according to Minniti \& Zijlstra 1997) for objects with $23.5 \leq m_{[\mathrm{O} \text { III }]} \leq 24.5$ for NGC 185 and NGC 205, and $24.5 \leq m_{[\mathrm{O} \text { III }]} \leq 25.5$ for NGC 147. For these magnitudes, the recovery rate decreases in the innermost regions of the galaxies due to the higher background. For brighter objects, the recovery rate is $100 \%$ at any galactocentric distance, except for the innermost 60 arcsec where however it decreases only by some $25 \%$. The difference between NGC 147 and the other 


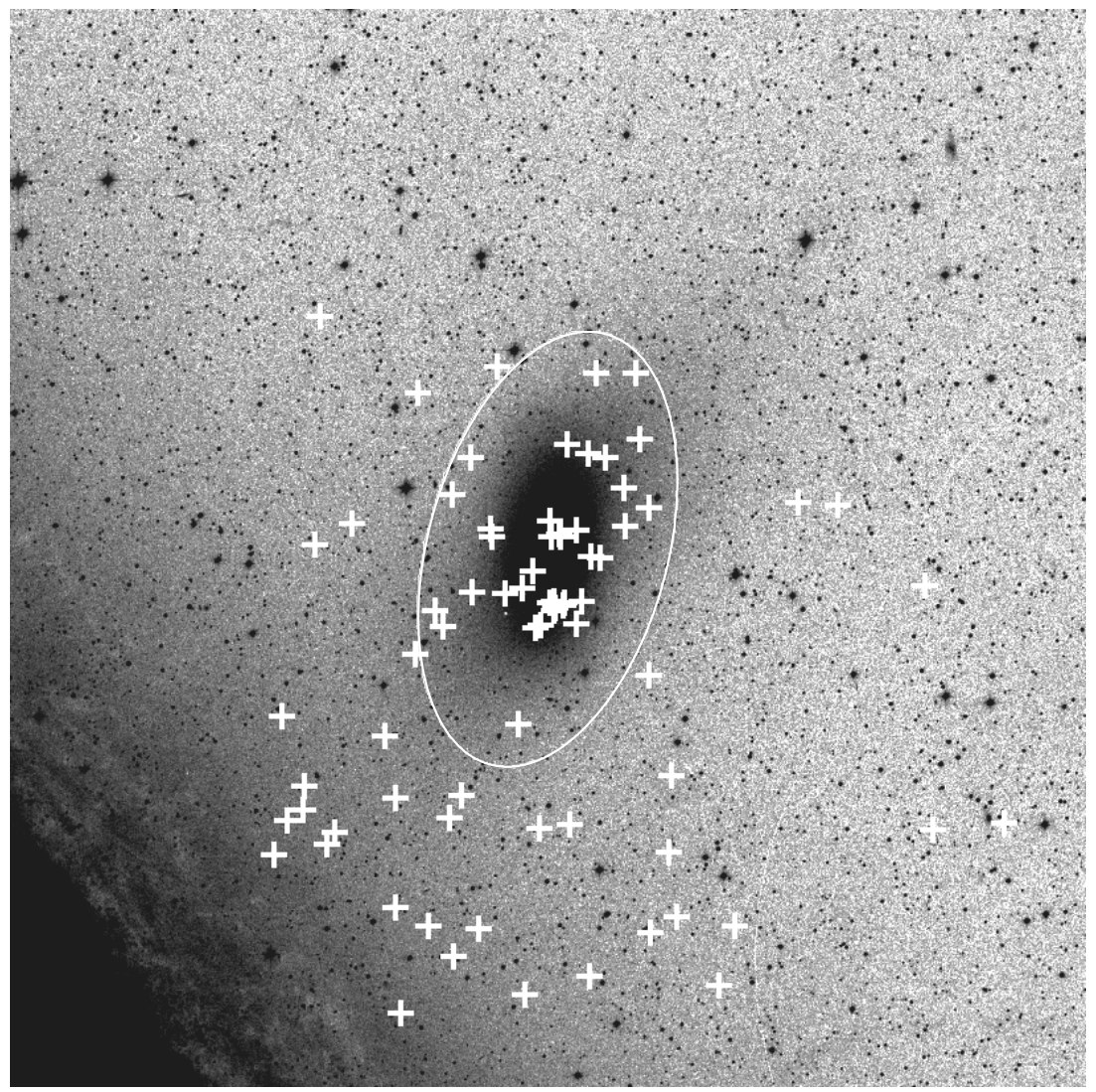

Fig. 4. Digitized Sky Survey image of an area of 50' $\times 50^{\prime}$ around NGC 205 (a portion of the disk of M 31 is visible in the bottom-left corner of the field). North is at the top, East to the left. Candidate PNe are marked with crosses. The ellipse at 1.5 tidal radii is indicated.

two galaxies is due to the fact that the former was observed in better seeing conditions, and (less important) that it has a lower surface brightness than the latter ones, both in terms of its stellar and gas background emission.

We have also measured the [O III] and $\mathrm{H} \alpha$ fluxes of the artificial stars in the same way as we have done for our candidate $\mathrm{PNe}$, and compared the measured fluxes with the input ones, confirming the magnitude of the photometric errors listed at the end of Sect. 3.1.

The PN specific luminosity rate for the three galaxies was estimated in the following way. We have adopted the total absolute $\mathrm{V}$ luminosity of the galaxies from vdB00, where distances of $660 \mathrm{kpc}$ for NGC 147 and NGC 185, and of $760 \mathrm{kpc}$ for NGC 205, are assumed. The bolometric correction is taken from Buzzoni \& Arnaboldi (2004). The observed number of PNe was then extrapolated to 8 mag down the bright end of the PNLF using its analytical description (Ciardullo et al. 1989). This allows us to compute the PN specific luminosity rate, that is found to be $1.4 \pm 0.3 \times 10^{-7} \mathrm{PNe} L_{\odot \text { bol }}^{-1}$ for both NGC 147 and NGC 185, and $2.3 \pm 0.2 \times 10^{-7}$ PNe $L_{\odot \text { bol }}^{-1}$ for NGC 205 . Scaled to our Galaxy, this would imply a total number of 5300 to 8700 Galactic PNe, in agreement with other recent estimates (e.g. Jacoby \& De Marco 2002). Note, however, that the extrapolation to faint magnitudes using the double-exponential formula of Ciardullo et al. (1989) may overestimate the total number of PNe by up to a factor of two (for the completeness limit of the observations presented in this paper), if the deep
PNe counts in the SMC presented by Jacoby (2004) are representative of the faint end of the PNLF for most galaxies. On the other hand, the PNLF of the bulge of M 31 seems to follow the empirical law, so the issue remains open and should be further investigated by much deeper surveys in the LG.

The number of PNe found in NGC 147, NGC 185, and NGC 205 also nicely fits into the scaling relation between the absolute $\mathrm{V}$ luminosity of LG galaxies and their PN population size (Magrini et al. 2003; Corradi \& Magrini 2004).

\subsection{The luminosity function of PNe in NGC 205}

In spite of the relatively small number of PNe, we have constructed the PNLF for the $35 \mathrm{PNe}$ located within 1.5 tidal radii from the centre of NGC 205 (see discussion in Sect. 4.3). This is presented in Fig. 6. The Eddington formula (Eddington 1913) was applied to correct for the effects of observational errors on the number of PNe in each bin. The resulting luminosity function within the completeness limit was then fit to the "universal" PNLF $N(m) \propto A \mathrm{e}^{0.307(m)}\left(1-\mathrm{e}^{3\left(m_{*}-m\right)}\right)$, taking into account both errors in $x$ (associated with the finite width of the histogram bins) and in $y$ (statistical errors). The derived distance modulus is $24.65 \pm 0.21$, adopting the absolute value of $M_{*}=-4.48$ (Ciardullo et al. 2002), and after correcting for foreground reddening using $E(B-V)=0.035$ (Burstein $\&$ Heiles 1984). The error on the distance modulus was computed combining the error derived from the fit with the errors 


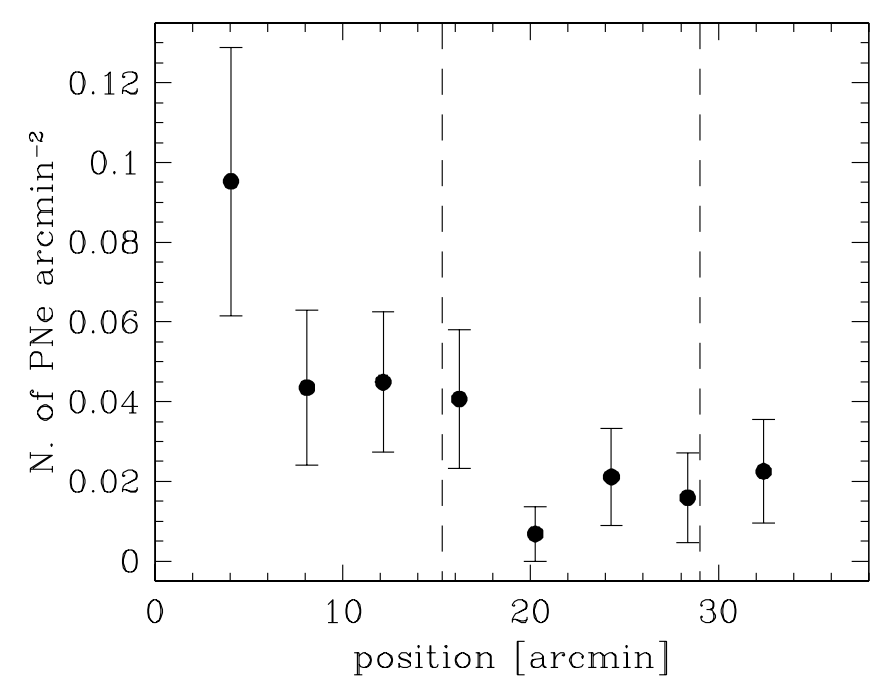

Fig. 5. The PN surface density at increasing distances from M 31 in strips parallel to its major axis, and outside 1.5 tidal radii from the centre of NGC 205 (see text). The zero point of the $x$-axis corresponds to the inner limit of our observed field. The dashed lines indicate the range of distances within which NGC 205 is located.

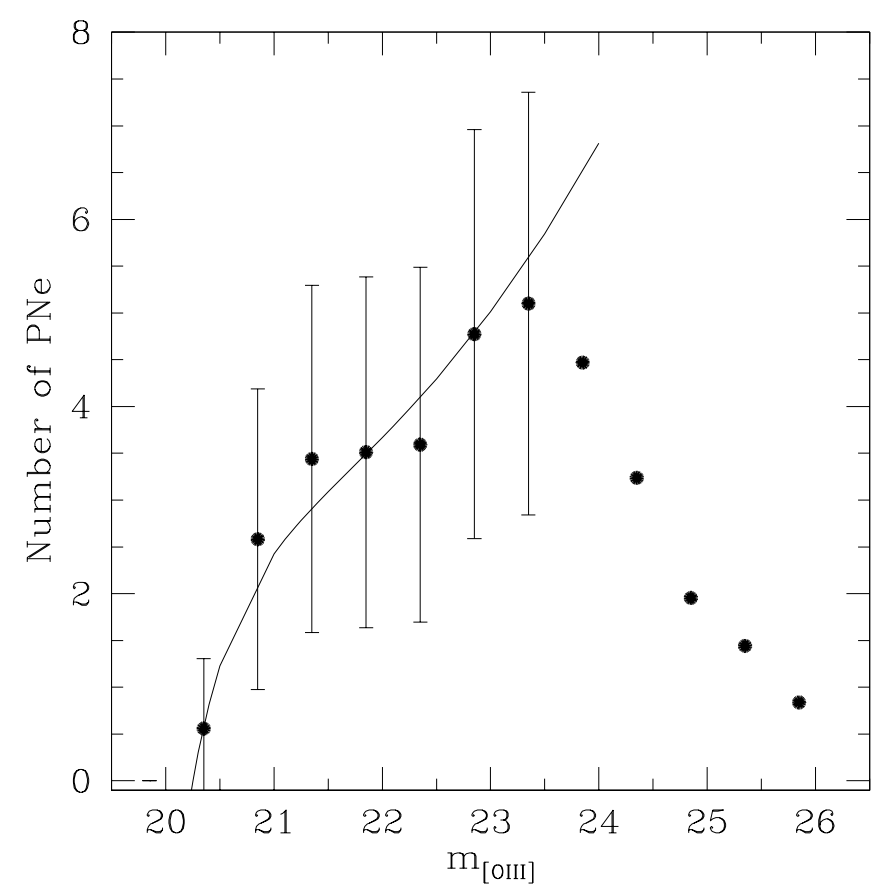

Fig. 6. The luminosity function of the 35 candidate $\mathrm{PNe}$ within 1.5 tidal radii from the centre of NGC 205. The fit down to the completeness limit of our images is indicated by the solid line.

on our photometric zero point and on the adopted extinction. The same distance modulus, within the errors, is obtained from the PNLF for objects located outside 1.5 tidal radii from the centre of NGC 205, presumably belonging to the halo and disc of M 31. This is also in agreement with the determination of Ciardullo et al. (1989) and, within the relatively large errors due to the small number of objects comprising the PNLF, with other determinations of the distance of NGC 205 (vdB00).

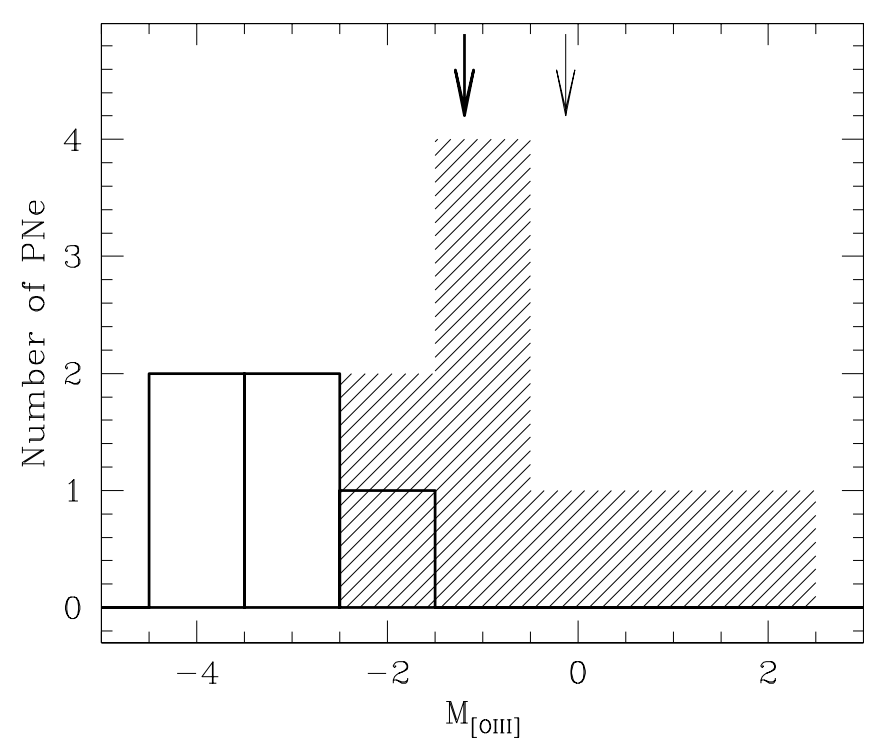

Fig. 7. Histogram of the absolute magnitudes of the candidate PNe in NGC 147 (dashed histogram) and NGC 185 (thick solid line). The completeness limits of our survey are indicated for each galaxy by the arrows in the upper part of the diagram.

\subsection{Age effects in the luminosity of PNe in NGC 185 and NGC 147?}

Figure 7 shows the histogram of the absolute [O III] magnitudes of the candidate PNe in NGC 147 and NGC 185, computed adopting a distance of $660 \mathrm{kpc}$ for both galaxies and a reddening $E(B-V)=0.17$ and 0.19 , respectively (vdB00). In spite of the small number of objects involved, there is some evidence that in [O III] the PNe in NGC 147 are systematically fainter than those of NGC 185 , which populate the bright end of the "universal" PNLF. Application of a 2-sample Kolmogorov-Smirnov test shows that indeed the two distributions are likely to be different at a significance level (probability of being wrong by saying that the two distributions are not drawn from the same population) smaller than $5 \%$. The same result holds even if we increase the distance modulus of NGC 147 by 0.4 mag (relative to NGC 185), as suggested by certain authors (cf. vdB00).

This difference might be related to the puzzling overall differences between the two galaxies. In fact, in spite of forming a bound (but not interacting) physical pair and having very similar morphological types, luminosities, and metallicities (vdB00), NGC 147 and NGC 185 are strikingly different in terms of their gas and dust content, likely as a consequence of a different evolution. NGC 147 is gas free (Sage et al. 1998), and Fig. 8 in Mateo (1998) would suggest that no star formation has occurred in this galaxy in the last $2-3 \mathrm{Gyr}$. On the other hand, the detailed photometric study of NGC 185 by Martinez-Delgado et al. (1999) derived a significant star formation in the last few Gyr in the inner region of this galaxy (see also Battinelli \& Demers 2004), and specifically within an elliptical isophote of semi-major axis of $\sim 240$ arcsec, where all our 5 candidate PNe are located.

The bright PNe in the inner regions of NGC 185 might therefore be the product of stars formed in the last few Gyr, 
while the absence of such bright PNe in NGC 147 would be the consequence of the absence of such a relatively young stellar population. This is an important issue, as recent theoretical modelling of the PNLF (Marigo et al. 2004) has indeed predicted its bright cutoff to be extremely sensitive to the time when star formation has stopped in a galaxy. According to Marigo et al. (2004), the effect would be particularly important if the last burst of star formation occurred more than 1-2 Gyr ago, which is the lifetime of stars with initial masses around 2-2.5 $M_{\odot}$ which are predicted to be those populating the bright end of the PNLF. Thus our data on NGC 147 and NGC 185 seem to provide some evidence for the age effects predicted by Marigo et al. (2004). Note also that the average luminosity difference between the PNe of the two galaxies is slightly smaller if the $\mathrm{H} \alpha$ fluxes, rather than the [O III] ones, are considered, a fact that is also predicted by the models of Marigo and collaborators (Girardi, private communication).

Our result, however, should be taken with extreme caution because of the low number statistics of the PN samples of NGC 147 and NGC 185. A better knowledge of the recent star formation history of NGC 147 would also be desirable. In addition, note that other (but less detailed) simulations of the PNLF show a much milder age effect (e.g. Méndez \& Soffner 1997). Moreover, the strong variation of the bright cutoff magnitude of the PNLF predicted by Marigo et al. (2004) has not been observed in the wide variety of galaxian types (spiral discs, bulges, ellipticals, irregulars) observed so far (cf. Ciardullo et al. 2002).

\section{Summary}

We have presented the result of our search for PNe in the three spheroidal galaxies NGC 147, NGC 185 and NGC 205. This increases the number of candidate PNe known in these galaxies, and provides a valuable resource for follow-up spectroscopy which will allow us to tackle some of the present problems in our understanding of these spheroidal galaxies. New abundances measurements (to improve the works by e.g. Richer \& McCall 1995) are in fact needed to shed light into the discrepancy between the metallicity determination from different authors in NGC 205, or to investigate further the difference and similarities between NGC 147 and NGC 185.

For these latter two galaxies, the present data show some evidence for age effects on the magnitude of the brightest PNe along the lines of the theoretical predictions by Marigo et al. (2004), the older stellar population of NGC 147 producing fainter PNe than the younger population of NGC 185 . This potentially important result is however weakened by low number of PNe found in these small galaxies, as well as in the general difficulty of determining precisely their star formation history in the last few Gyr. Testing the possible existence of such an age effect in other galaxies (within or outside the LG) would be desirable, but it is likely not be an easy task, as the star formation history is found to be significantly different from galaxy to galaxy, even in systems of the same morphological type, mass and metallicity.

Acknowledgements. We thank Dr. Rino Bandiera for his suggestions on the fitting method of the PNLF of NGC 205. The LGC data are publically available through the Isaac Newton Group Wide Field Camera Survey Programme.

\section{References}

Arnaboldi, M., Freeman, K. C., Okamura, S., et al. 2003, AJ, 125, 514 Battinelli, P., \& Demers, S. 2004, A\&A, 417, 479

Burstein, D., \& Heiles, C. 1984, ApJS, 54, 33

Buzzoni, A., \& Arnaboldi, M. 2004, in Planetary Nebulae beyond the Milky Way, ESO Astrophys. Symp., ed. J. R. Walsh, \& L. Stangehllini (Springer-Verlag), in press

Ciardullo, R., Jacoby, G. H., Ford, H. C., \& Neill, J. D. 1989, ApJ, 339, 53

Ciardullo, R., \& Jacoby, G. H. 1992, ApJ, 338, 268

Ciardullo, R., Feldmeier, J. J., Jacoby, G. H., et al. 2002, ApJ, 577, 31

Corradi, R. L. M., \& Magrini, L. 2004, in Planetary Nebulae beyond the Milky Way, ESO Astrophys. Symp., ed. J. R. Walsh, \& L. Stangehllini (Springer-Verlag), submitted

Eddington, A. S. 1913, MNRAS, 73, 359

Feldmeier, JJ., Ciardullo, R., Jacoby, G. H., \& Durrell, P. R. 2003, ApJS, 145, 65

Ford, H. C., Jenner, D. C., \& Epps, H. W. 1973, AJ, 183, 73

Ford, H. C., Jacoby, G., \& Jenner, D. C. 1977, ApJ, 213, 18

Ford, H. C., \& Jacoby, G. H. 1978, ApJS, 38, 351

Holmberg, E. 1958, Lund Medd. Astron. Obs. Ser. II, 136, 1

Irwin, M., \& Lewis, J. 2001, New AR 45, 105

Jacoby, G. H. 1989, ApJ, 339, 39

Jacoby, G. H. 2004, in Planetary Nebulae beyond the Milky Way, ESO Astrophysics Symposia, ed. J. R. Walsh, \& L. Stangehllini (Springer-Verlag), in press

Jacoby, G. H., \& De Marco, O. 2002, AJ, 123, 269

Leisy, P., Corradi, R., Davenport, et al. 2004, A\&A, in preparation

Magrini, L., Corradi, R. L. M., Walton, N. A., et al. 2002, A\&A, 386, 869 (M02)

Magrini, L., Corradi, R. L. M., Greimel, R., et al. 2003, A\&A, 407, 51

Magrini, L., Corradi, R. L. M., Greimel, R., et al. 2004, A\&A, in preparation

Marigo, P., Girardi, L., Weiss, A., Groenewegen, M. A. T., \& Chiosi, C. 2004, A\&A, submitted

Martinez-Delgado, D., Aparicio, A., \& Gallart, C. 1999, AJ, 118, 2229

Mateo, M. 1998, ARA\&A, 36, 435

Méndez, R. H., \& Soffner, T. 1997, 321, 898

Merrett, H. R., Kuijken, K., Merrifield, M. R., et al. 2003, MNRAS, 346, L62

Meyssonnier, N., Lequeux, J., \& Azzopardi, M. 1993, A\&AS, 102, 251

Minniti, D., \& Zijlstra, A. A. 1997, AJ, 114, 147

Monet, D., Bird, A., Canzian, B., et al. 1998, USNO-A2.0, (U.S. Naval Observatory, Washington DC)

Oke, J. B. 1990, AJ, 99, 1621

Renzini, A., \& Buzzoni, A. 1986, in Spectral Evolution of Galaxies, ed. C. Chiosi, \& A. Renzini (Reidel), Ap. Space Sci. Lib., 122, 195

Richer, M. G., \& McCall, M. L. 1995, ApJ, 445, 642

Sage, L. J., Welch, G. A., \& Mitchell, G. F. 1998, ApJ, 507, 726

Stetson, P. B. 1994, PASP, 106, 250

van den Bergh, S. 2000, in The Galaxies of the Local Group (Cambridge: Cambridge University Press), 177 (vdB00)

Wright, S., Corradi, R. L. M., \& Perinotto, M. 2004, A\&A, submitted 